\title{
ANB, Wits and Molar Relationship, Do they correlate in Orthodontic Patients?
}

\author{
Al-Jabaa $\mathrm{AH}$ and Aldrees $\mathrm{AM}^{\star}$
}

Department of Pediatric Dentistry and Orthodontics, College of Dentistry, King Saud University, Riyadh, Saudi Arabia

*Corresponding author: Abdullah M Aldrees, Associate Professor, Division of Orthodontics, Department of Pediatric Dentistry and Orthodontics, College of Dentistry, King Saud University, P.O. Box 60169, Riyadh 11545, Saudi Arabia, Tel: +966-11-8051975; Fax: +966-11-4694793; E-mail: amaldrees@ksu.edu.sa

Rec date: Mar 07, 2014; Acc date: May 26, 2014; Pub date: May 28, 2014

Copyright: (c) 2014 Al-Jabaa and Aldrees. This is an open-access article distributed under the terms of the Creative Commons Attribution License, which permits unrestricted use, distribution, and reproduction in any medium, provided the original author and source are credited.

\begin{abstract}
Objective: To determine the association between the anteroposterior relationship of the dental arch and the jawbase in a random sample of orthodontic patients.

Materials and Methods: 478 pretreatment lateral cephalometric radiographs and study models were evaluated. Cephalometric analysis using Dolphin software to measure the ANB angle and Wits appraisal was performed, and Angle's classification was evaluated.

Results: The agreement of the sagittal classification of the dental arch was higher with the anteroposterior relationship of the jaw-base using ANB angle (57.7\%) than with Wits appraisal (43.7\%). Agreement of molar relation and both ANB and Wits was higher in Class I cases (79.5\% and $84 \%$ respectively). The correlation coefficient value between ANB and Wits was 0.727 . A statistical significant regression between ANB and Wits was found.

Conclusions: It is evident that the Angle classification of occlusion will not reveal the full picture of the dentofacial deformity, and the three methods of anteroposterior classification agree in only one out of every three individuals.
\end{abstract}

Keywords: Wits; ANB; Cephalometric; Angle Molar Relation

\section{Introduction}

The relationships between craniofacial morphology and malocclusion have long been of interest to orthodontists. Angle's classification of malocclusion is based on the mesiodistal relationship between the upper and lower dental arches [1]. Subsequent studies revealed that the dental arch relationship is largely affected by the facial skeleton upon which the teeth are invested [2-4]. Bjork reported that local changes confined to the teeth can exercise a limited effect on the discrepancy in the relationship between the upper and lower dental arches, whereas facial or cranial configurations may produce marked effects $[2,3]$. Few studies have investigated the relationship between the anteroposterior dental arch and jaw-base relationships. Taylor pointed out that ANB difference is not always a true indication of the apical base relationship. Certain changes in the relative position of Nasion to points A and B influence the degree to which ANB fails to reflect apical base relationship [5]. In Finnish children, Haavikko and Hele reported that every Angle class includes a large proportion of atypical facial types [6]. The anteroposterior dysplasia indicator (APDI) was found by Kim to be the cephalometric measurements that would produce the highest correlation against the displacement of the molar relationships [7]. Milacic and Markovic reported that $85 \%$ of Angle Class III individuals had identical dental and skeletal relationships [8]. Glisic and Jaksic investigated Schwarz's and Steiner's cephalometric analyses, and they found that both methods agreed on the same skeletal relationship in only $58.6 \%$ of all investigated cases [9].
Zhou et al. found that the anteroposterior relationship of the dental arch and the jaw-base fail to match in at least one out of every three individuals, and the linear measurement of anteroposterior jaw-base relationships is a more valid reflection of the dental arch relationship than angular measurements [10].

Within the orthodontic community, the issue of whether the anteroposterior dental arch correlates with the jaw-base relationship and thus whether more than one assessment needs to be included in orthodontic diagnosis and treatment planning is still not clearly determined. This study aimed to investigate the association between the anteroposterior dental arch and the jaw-base relationships evaluated by two widely used cephalometric measurements in a sample of orthodontic patients.

\section{Materials and Methods}

Pre-treatment dental casts and lateral cephalometric radiographs were obtained randomly after searching through 3400 files from the archive of the Orthodontic Clinic at the College of Dentistry, King Saud University. 478 patients who fulfilled the following inclusion criteria formed the sample of the study:

All subjects should be in the permanent dentition stage.

Complete pre-treatment records taken within a period of 6 months.

Patients with syndromes, developmental anomalies, extraction of any permanent teeth, and history of a previous orthodontic treatment, prosthodontic treatment or trauma to any tooth before the commencement of orthodontic treatment were excluded. 
Page 2 of 5

One trained examiner assessed the molar relationship (anteroposterior dental arch relationship) on the basis of Angle's definition. Molar Class I was defined as occurring when the mesiobuccal cusp of the upper first molar occludes with the mesiobuccal groove of the lower first molar or within the range of less than half a cusp width anteriorly or posteriorly. Cases that were determined to have an asymmetric molar relationship were excluded. Cephalometric radiographs were digitized and analyzed using Dolphin Imaging 10.0 software (Dolphin Imaging and Management Solutions, Chatsworth, California) and the following measurements were obtained:

ANB angle [11]: Subjects were classified into different malocclusion groups based on the following criteria:

\section{Skeletal Class I: $0^{\circ}$ to $4^{\circ}$}

Skeletal Class II: $>4^{\circ}$

Skeletal Class III: $<0^{\circ}[12-14]$

Wits appraisal: The sample size, mean and standard deviation derived from the Jacobson study were used to pool the male and female measurements [15]. Accordingly, the following ranges of the skeletal classes were defined:

\section{Skeletal Class I: -1.8 to $0.8 \mathrm{~mm}$}

Skeletal Class II: $>0.8 \mathrm{~mm}$

Skeletal Class III: $<-1.8 \mathrm{~mm}$

\section{Analysis of the Data}

Data were evaluated using Statistical Package Software System, version 13 (SPSS Inc., Chicago, Illinois), and the level of significance was set at $\mathrm{p}<0.05$. The following tests were used:

Error of Method: for intra-examiner reliability, measurements were compared statistically using paired t-test and coefficient of reliability, and no statistically significant differences were observed between the first and the second readings of ANB and Wits using paired t-test $(p>0.05)$. The coefficient of reliability was 0.970 for the two ANB and Wits measurements.

Association between the anteroposterior relationship of the dental arch and the anteroposterior relationship of the jaw-base:

Chi-square was used to evaluate the presence of an association.

Kappa statistics was used to measure the association.

One-way ANOVA: to determine the differences in the mean values of ANB and Wits across molar classes.

Correlation and regression between ANB and Wits.

\section{Results}

\section{Agreement between molar classes and ANB skeletal classes}

Cross tabulation chi-square showed that the distribution of different molar classes among the skeletal classes was uneven $(\mathrm{p}<0.0001)$. Kappa statistic showed that there is a statistically significant fair agreement between molar classes and ANB skeletal classes (Table 1). The agreement was significant in male and female groups. Among males $(n=226)$, the agreement between molar classes and ANB skeletal classes was found in $57.5 \%$ of the patients, while in females $(n=252)$, the agreement was found in $57.9 \%$.

\begin{tabular}{|c|c|c|c|c|c|c|}
\hline Molar & ANB & & & Total & \multirow{2}{*}{$\begin{array}{l}\text { chi-square } \\
\text { (p-value) }\end{array}$} & \multirow{2}{*}{$\begin{array}{l}\text { Kappa (k) } \\
\text { Statistic } \\
\text { (p-value) }\end{array}$} \\
\hline & Class I & Class II & $\begin{array}{l}\text { Class } \\
\text { III }\end{array}$ & & & \\
\hline Class I & 198 & 127 & 19 & 344 & \multirow{8}{*}{$(p<0.0001)^{*}$} & \multirow{8}{*}{$\begin{array}{l}0.219 \\
(p<0.0001)^{*}\end{array}$} \\
\hline$\%$ & $41.40 \%$ & $26.70 \%$ & $3.90 \%$ & $72 \%$ & & \\
\hline Class II & 34 & 58 & 1 & 93 & & \\
\hline$\%$ & $7.10 \%$ & $12.10 \%$ & $0.21 \%$ & $19.50 \%$ & & \\
\hline Class III & 17 & 4 & 20 & 41 & & \\
\hline$\%$ & $3.50 \%$ & $0.84 \%$ & $4.20 \%$ & $8.50 \%$ & & \\
\hline \multirow[t]{2}{*}{ Total } & 249 & 189 & 40 & 478 & & \\
\hline & $52.10 \%$ & $39.50 \%$ & $8.40 \%$ & $100 \%$ & & \\
\hline
\end{tabular}

Table 1: Agreement between anteroposterior molar relation and ANB angle

\section{Agreement between molar classes and Wits appraisal classes}

Chi-square and Kappa statistic showed that there was a statistically significant fair agreement between molar classes and Wits skeletal classes $(\mathrm{p}<0.0001)($ Table 2$)$. This agreement was also evident in both males and females. Among males, the agreement was found in $44.7 \%$ of the patients, while in females, it was present in $42.8 \%$. Twenty-nine percent of the patients had identical anteroposterior classes in dental arch and jaw-base relationships determined by ANB and Wits analyses (69 Class I, 49 Class II, and 20 Class III).

\begin{tabular}{|c|c|c|c|c|c|c|}
\hline Molar & Wits & & & Total & \multirow{2}{*}{$\begin{array}{l}\text { chi-square } \\
\text { (p-value) }\end{array}$} & \multirow{2}{*}{$\begin{array}{l}\text { Kappa }(\mathbf{k}) \\
\text { Statistic } \\
\text { (p-value) }\end{array}$} \\
\hline & Class I & Class II & $\begin{array}{l}\text { Class } \\
\text { III }\end{array}$ & & & \\
\hline Class I & 110 & 102 & 132 & 344 & \multirow{8}{*}{$(p<0.0001)^{*}$} & \multirow{8}{*}{$\begin{array}{l}0.198 \\
(p<0.0001)^{*}\end{array}$} \\
\hline$\%$ & $23 \%$ & $21.30 \%$ & $27.60 \%$ & $72 \%$ & & \\
\hline Class II & 18 & 64 & 11 & 93 & & \\
\hline$\%$ & $3.80 \%$ & $13.40 \%$ & $2.30 \%$ & $19.50 \%$ & & \\
\hline Class III & 3 & 3 & 35 & 41 & & \\
\hline$\%$ & $0.63 \%$ & $0.63 \%$ & $7.30 \%$ & $8.50 \%$ & & \\
\hline \multirow[t]{2}{*}{ Total } & 131 & 169 & 178 & 478 & & \\
\hline & $27.40 \%$ & $35.40 \%$ & $37.20 \%$ & $100 \%$ & & \\
\hline
\end{tabular}

Table 2: Agreement between anteroposterior molar relation and Wits appraisal 
Page 3 of 5

\section{Comparing the mean values of ANB and Wits across different molar classes}

One-way ANOVA showed that there were statistically significant differences in the mean values of ANB and Wits across the molar classes $(\mathrm{p}<0.0001)$. Post Hoc tests revealed that the ANB and Wits mean values were statistically significantly higher in Class II molar relation than in other molar classes (Table 3).

\begin{tabular}{|l|l|l|l|l|l|}
\hline Skeletal Relation & \multicolumn{2}{|l|}{ Molar Relation } & & Total & ANOVA \\
\hline & Class I & Class II & Class III & & (p-value) \\
\hline & $(\mathbf{n}=\mathbf{3 4 4})$ & $(\mathbf{n}=\mathbf{9 3})$ & $\mathbf{( n = 4 1 )}$ & & \\
\hline ANB $\left(^{\circ}\right)$ & $3.305 \pm 2.172$ & $4.647 \pm 2.254$ & $-0.068 \pm 3.403$ & $3.277 \pm 2.318$ & $(\mathbf{p}<0.0001)^{*}$ \\
\hline Wits $(\mathrm{mm})$ & $-0.888 \pm 3.088$ & $2.381 \pm 3.261$ & $-6.212 \pm 5.124$ & $-0.710 \pm 3.343$ & $(\mathbf{p}<0.0001)^{*}$ \\
\hline${ }^{*}$ 'Statistically significant & & & & \\
\hline
\end{tabular}

Table 3: ANB angle and Wits appraisal values across molar classes (means $\pm \mathrm{SD}$ )

\section{Correlation of ANB and Wits values across molar classes}

Pearson correlation test was used to determine the correlation of ANB and Wits values across the molar classes, and all correlation coefficients were highly statistically significant:

In Class I molar relation: $\mathrm{r}$-value $=0.743$.

In Class II molar relation: $\mathrm{r}$-value $=0.758$.

In Class III molar relation: $r$-value $=0.746$.

The overall correlation coefficient between ANB and Wits was 0.727 (Figure 1).

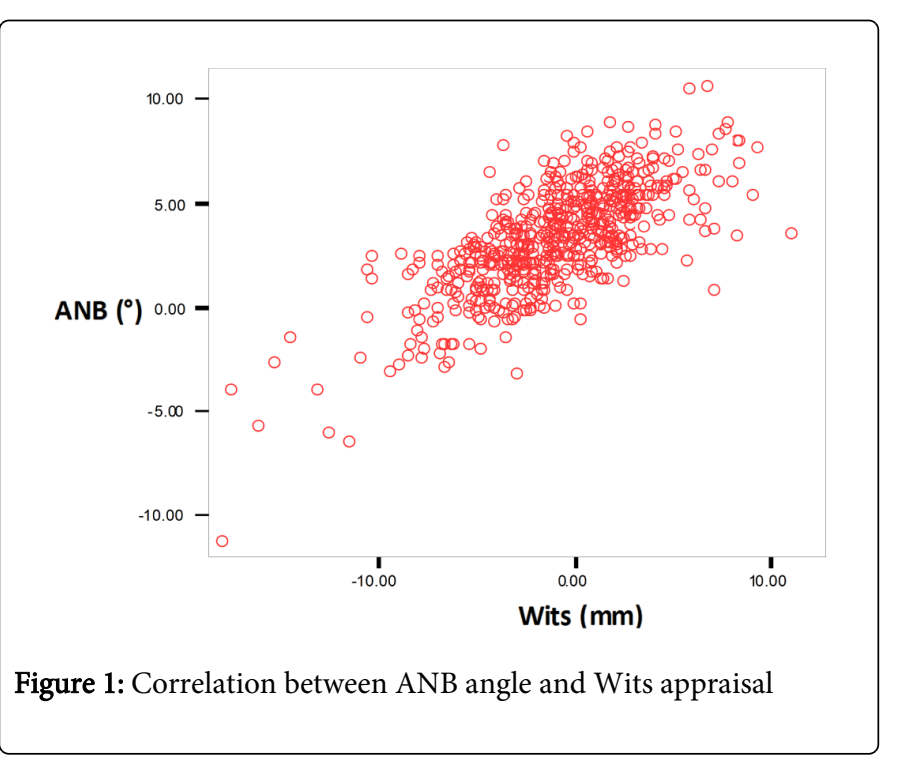

\section{Regression between ANB and Wits}

Regression test showed that there was a statistically significant relationship $(\mathrm{p}<0.0001)$ between ANB and Wits values, in which $55.2 \%$ change in ANB values can be explained by Wits.

\section{Discussion}

The use of cephalometric radiography in orthodontic research and clinical practice adds an important value to the dental records in precisely measuring dentofacial structures and evaluating treatment outcome. Conflicting results that may be brought by cephalometric assessments and lack of agreement with dental cast classifications contribute to the low 'weight' of lateral cephalometry in the therapeutic decision-making process by the orthodontist [16-18]. This study revealed that Angle classification of occlusion is not always a true indicator of the underlying skeletal deformity, and it reports areas of agreement of the popular methods for assessing dentoalveolar sagittal relationship.

In the present study, the agreement between molar relations and ANB angle was 57.7\%, however, Milacic and Markovic reported a higher concordance between molar relation and skeletal relation using ANB angle (75\%) [8]. Zhou et al. found that the anteroposterior dental arch relation did coincide with the jaw-base relationships, as expressed by the ANB angle in $61 \%$, a percentage closely matching our results $[8,10]$. A possible explanation for the difference between our results and those reported by Milacic and Markovic could lie in their sample which included subjects in the mixed dentition stage. First permanent molars tend to assume an end-to-end relationship when they first erupt in the mixed dentition before their transition into Class I relationship due to the differential mandibular growth and the mesial shift into the leeway space [19].This dental relationship along with relatively deficient mandible before it gradually catches up may have affected the outcome Milacic and Markovic reported [8].

This study showed an agreement between molar relationship and Wits appraisal that was lower than the percentage reported by Zhou et al. (43.7\% vs. $67 \%$ ) [10]. These differences might be due to the methodology employed by the two studies. Zhou et al. reported that they classified the skeletal malocclusion into Class I if it falls within the range of the mean value of the sample \pm one standard deviation. Thus, the range of skeletal Class I in their study using Wits was significantly wide $(-7.0$ to $-1.6 \mathrm{~mm})$ [10]. Our criteria in classifying skeletal malocclusion were consistent with Wits normal ranges, and it should represent more closely the reported distribution of the skeletal classification. The reported concordance between ANB and Angle molar classification was higher in Class I cases, this was in agreement with Zhou et al. [10]. In addition, the agreement percentages in Class II and Class III malocclusions were also similar in the two studies.

The least agreement in our results was between Class III molar relation and skeletal evaluation using Wits appraisal. This might be explained by the fact that molar relation is affected by several factors, 
including mandibular plane angle. It has been shown that increased mandibular plane angle leads to a more posteriorly located lower first molars [20]. In addition, it has been illustrated previously that Saudi patients have a tendency towards an increased mandibular plane angle [21]. This could lead to a skeletal evaluation of a Class III relationship by Wits appraisal, but due to the increased mandibular plane angle, the lower first molars will be located more posteriorly and a malocclusion of Class I or Class II molar relation will result. In addition, in our sample a few Class III molar relations were included and this may have contributed to the low agreement between Class III molar relation and Wits classification. ANB angle is also strongly influenced by geometric factors, and the absolute value of ANB increases with the length of the vertical distance between the points $A$ and B [22]. This may have contributed to the larger percentages of cases diagnosed with Class I and II malocclusion in the sample compared to Wits that classified more patients as Class III. It is thus evident that the Angle classification of occlusion, which is based on the dental arch relationship alone, will not reveal the full picture of the dentofacial deformity, not even in the sagittal plane. This was in accordance with other studies $[6,8,10]$.

Orthodontic patient samples can be heavily biased. It was reported from the Milacic and Markovic study that dental arch and skeletal Class III classification coincide in $85 \%$ of patients, whereas results from our study, Haavikko and Helle, and Zhou et al. demonstrated that the agreement between ANB and Class III molar relation is far less. This might be due to the small number of Class III patients included in the Milacic and Markovic study $[6,8,10]$.

We found that ANB and Wits mean values were statistically significantly higher in Class II molar relation than in the other molar relationships. This follows the logical agreement between the skeletal relationship and the molar relation since that high ANB and Wits measurements usually indicate Class II relationship.

Our results revealed that the correlation coefficient ( $r$-value), which represents the probability to predict the variables from one another, was relatively moderate between ANB and Wits values. Horowitz and Hixon have stated that "Correlation coefficient of less than $r=0.7$ or $\mathrm{r}=0.8$ has little predictive value when compared to an individual. A correlation coefficient of $r=0.7$ means that less than one half of the total variation can be eliminated in prediction" [23]. This means that a certain value of the ANB angle may be associated with many values of the Wits appraisal, and thus the prediction between variables is moderate, and the association between the ANB angle and Wits appraisal did not differ among the various classes of dental arch relationship. These results were in agreement with Zhou et al and Wellens $[10,24]$. Conflicting results between ANB and Wits is a result of shortcomings in both methods of measurement. While ANB is affected by facial height, jaw inclination, vertical development of the face and anteroposterior and vertical position of Nasion, Wits is affected by occlusal plane inclination and has been identified as the measurement with the greatest coefficient of variability among the methods of cephalometric analysis for assessing sagittal jaw relationship [25-27]. To help clinicians arrive at a more accurate diagnosis of the case, the use of both the ANB angle and the Wits appraisal in addition to the other diagnostic criteria, including the molar relationship and the soft tissue features, is recommended. Improved agreements between these two measurements could also result from optimizing the cut-off points of the skeletal classifications [28], and 'fitting' a template on the patient's digitized landmarks to minimize the geometric distortion [24].
Our results revealed a statistically significant regression between ANB angle and Wits appraisal which was in agreement with Hurmerinta et al. and Zhou et al., however, Hurmerinta et al. reported a lower R2 value $[10,29]$. This might be due to age differences because their study included patients with primary dentition [29]. Bishara et al. reported in a longitudinal study that it was determined statistically that ANB is a function of age and Wits is not. By virtue of this fact, it can be said that ANB and Wits change differently over time, which will affect their correlation [27].

\section{Conclusions}

The agreement between the molar relationship and ANB angle was higher than the agreement between the molar relationship and Wits appraisal.

The agreement between the molar relationship and both ANB and Wits was higher in Class I cases followed by Class II then Class III.

A relatively moderate correlation coefficient value between ANB and Wits is evident, and a proportion of $55.2 \%$ change in ANB values could be explained by Wits.

\section{References}

1. Angle E (1899) Classification of malocclusion. Dental Cosmos 41: 248-264.

2. Bjork A (1947) Face in profile-an anthropological x-ray investigation on Swedish children and conscripts. Svensk Tandlakaretidskrift 40: 180.

3. Bjork A (1951) The nature of facial prognathism and its relation to normal occlusion of the teeth. Am J Orthod 37: 106-124.

4. Enlow D, Kuroda T, Lewis A (1971) The morphological and morphogenetic basis for craniofacial form and pattern. Angle Orthod 41: 161-188.

5. Taylor CM (1969) Changes in the relationship of nasion, point A, and point B and the effect upon ANB. Am J Orthod 56:143-163.

6. Haavikko K, Helle A (1974) A cephalometric study of facial types and occlusion classes and relation between them in Finnish children. Proc Finn Dent Soc 70: 99-106.

7. Kim YH, Vietas JJ (1978) Anteroposterior dysplasia indicator: an adjunct to cephalometric differential diagnosis. Am J Orthod 73: 619-633.

8. Milacic M, Markovic M (1983) A comparative occlusal and cephalometric study of dental and skeletal anteroposterior relationships. Br J Orthod 10: 53-54.

9. Glisic B, Jaksic N (1990) [Variations of sagittal skeletal relationships in cases with normal occlusion]. Bilt Udruz Ortodonata Jugosl 23: 87-91.

10. Zhou L, Mok CW, Hagg U, McGrath C, Bendeus M, et al. (2008) Anteroposterior dental arch and jaw-base relationships in a population sample. Angle Orthod 78: 1023-1029.

11. Steiner CC (1953) Cephalometrics for you and me. Am J Orthod 39: 729-755.

12. Alkofide EA (2007) The shape and size of the sella turcica in skeletal Class I, Class II, and Class III Saudi subjects. Eur J Orthod 29: 457-463.

13. Cha BK, Kim CH, Baek SH (2007) Skeletal sagittal and vertical facial types and electromyographic activity of the masticatory muscle. Angle Orthod 77: 463-470.

14. Uslu O, Akcam MO, Evirgen S, Cebeci I (2009) Prevalence of dental anomalies in various malocclusions. Am J Orthod Dentofacial Orthop 135: 328-335.

15. Jacobson A (1975) The "Wits" appraisal of jaw disharmony. Am J Orthod 67: 125-138.

16. Atchison KA, Luke LS, White SC (1991) Contribution of pretreatment radiographs to orthodontists' decision making. Oral Surg Oral Med Oral Pathol 71: 238-245. 
Citation: Al-Jabaa AH, Aldrees AM (2014) ANB, Wits and Molar Relationship, Do they correlate in Orthodontic Patients?. Dentistry 4: 240. doi:

Page 5 of 5

17. Han UK, Vig KW, Weintraub JA, Vig PS, Kowalski CJ (1991) Consistency of orthodontic treatment decisions relative to diagnostic records. Am J Orthod Dentofacial Orthop 100: 212-219.

18. Hansen K, Bondemark L (2001) The influence of lateral head radiographs in orthodontic diagnosis and treatment planning. Eur J Orthod 23: 452-453.

19. Proffit W, Fields H and Sarver D (2007) Contemporary Orthodontics. Mosby, St Louis.

20. Andria LM, Leite LP, Dunlap AM, Cooper EC, King LB (2007) Mandibular first molar relation to variable lower face skeletal components. Angle Orthod 77: 21-28.

21. Al-Jasser N (2005) Cephalometric evaluation using McNamara analysis in a sample of Saudi adults. Journal of the Pakistan Dental Association 14: 76-83.

22. Sadat-Khonsari R, Dathe H, Knosel M, Hahn W, Kubein-Meesenburg D, et al. (2009) Geometric influence of the sagittal and vertical apical base relationship on the ANB angle. J Orofac Orthop 70: 152-158.

23. Horowitz S and Hixon EH (1966) The nature of orthodontic diagnosis. Mosby, St Louis.
24. Wellens H (2009) Improving the concordance between various anteroposterior cephalometric measurements using Procrustes analysis. Eur J Orthod 31: 503-515.

25. Rotberg S, Fried N, Kane J, Shapiro E (1980) Predicting the "Wits" appraisal from the ANB angle. Am J Orthod 77: 636-642.

26. Bishara SE, Fahl JA, Peterson LC (1983) Longitudinal changes in the ANB angle and Wits appraisal: clinical implications. Am J Orthod 84: 133-139.

27. Gul e E, Fida M (2008) Pattern of malocclusion in orthodontic patients: a hospital based study. J Ayub Med Coll Abbottabad 20: 43-47.

28. Anderson G, Fields HW, Beck M, Chacon G, Vig KW (2006) Development of cephalometric norms using a unified facial and dental approach. Angle Orthod 76: 612-618.

29. Hurmerinta K, Rahkamo A, Haavikko K (1997) Comparison between cephalometric classification methods for sagittal jaw relationships. Eur J Oral Sci 105: 221-227. 